\title{
Affine group formulation of the Standard Model coupled to gravity
}

\author{
Ching-Yi Chow* \\ Department of Physics, National Cheng Kung University, Taiwan \\ Eyo Ita \\ Department of Physics, US Naval Academy, Annapolis Maryland \\ Chopin Sod \\ Dept. of Physics, National Cheng Kung University, Taiwan
}

\begin{abstract}
This work demonstrates that a complete description of the interaction of matter and all forces, gravitational and non-gravitational, can in fact be realized within a quantum affine algebraic framework. Using the affine group formalism, we construct elements of a physical Hilbert space for full, Lorentzian quantum gravity coupled to the Standard Model in four spacetime dimensions. Affine algebraic quantization of gravitation and matter on equal footing implies a fundamental uncertainty relation which is predicated upon a non-vanishing cosmological constant.
\end{abstract}

\section{INTRODUCTION}

In this work a new approach to the Wheeler-DeWitt constraint is presented. This approach will be based upon the affine group formalism, utilizing unitary irreducible representations of the group of transformations of the straight line $x \rightarrow a x+b$. Previous results by the present authors reveal a fundamental structure concealed within the Hamiltonian constraint of General Relativity (GR), which has provided insight into such an approach. The background and results needed for this paper are encapsulated in [1], 2], 3] and references therein. In [1] it was shown that the quantum Hamiltonian constraint of vacuum GR with cosmological constant can be written as an affine algebra involving the commutator of the imaginary part of the Chern-Simons funtional, $Q$, with the local volume operator $V(x)$, whose representations led directly to the construction of elements of a physical Hilbert space for gravity respecting the intital value constraints. The classical aspects of these results were independently derived in [2], in the metric representation, from a different context. In [2] the Hamiltonian constraint was written as a Poisson bracket of $Q$ with $V(x)$, which provided the insight of the conformal invariance of the real part of the Chern-Simons functional, a symmetry which becomes broken by its imaginary part. The necessary and sufficient condition for the invariance of this imaginary part with respect to infinitesimal rescalings of the spacetime metric is just that the spacetime metric satisfy the vacuum Einstein equations. In 3] the results of [2] were generalized to include the Higgs and the Yang-Mills fields, showing that the Hamiltonian constraint of the coupled theory can also be written as the Poisson bracket of a certain functional $G$ with the volume element. The kinematic constraints were not addressed.

In the present paper we will generalize these results, with the goal of quantization of the Standard Model coupled to gravity. One motivation stems from the observation that purely quantum gravitational effects presumably, by themselves, may not become directly observable until the Planck scale, several orders of magnitude beyond typical energies accessible to existing accelerators. A consistent treatment of the gravity coupling with matter, which quantizes all fields on an equal footing, should in-principle encode the quantum effects of gravity in this interaction. Conceivably, the imprints of gravity from the Planck scale could be encoded within observations which could be made on matter fields at everyday energy scales. This paper will constitute an attempt to bridge this gap via a proposal designed to respect the fundamental assumption of background independence in congruity with the axioms of quantum mechanics.

We will ultimately construct elements of a physical Hilbert space $\mathbf{H}_{P h y s}$, which respects the initial value constraints (Hamiltonian, diffeomorphism and Gauss' law constaints), for gravity coupled with matter fields. In order to carry out this goal we will need to put in place the following elements: (i) First, we will generalize the results of [3] to include coupling to Weyl spinor fields. Concurrently, we will adapt the matter Hamiltonians of [3] to density weight one form to bring them into congruity with [1]. This will be necessary in order to address the diffeomorphism-invariant aspects of the affine group representations. (ii) Once all contributions to the constraints have been placed on the same footing, then we will apply the formalism of [1] to the quantization of the coupled theory and to the construction of elements of the physical Hilbert space.

This paper proceeds as follows. In section 2 we establish some notations and conventions. Then we review the main results of [3] detailing the gravity-matter coupling for bosonic fields in the metric representation. The incorporation of spin $1 / 2$ fermions will entail the introduction of a vierbein, which we incorporate via the ADM triad-extrinsic curvature/Ashtekar variables. The purpose of section 2 is to establish the matter contributions to a functional $M$, 
whose role will be that of the dilator element comprising the affine Lie algebraic Hamiltonian constraint. In Section 3 we present a detailed analysis of the fermionic action, and the fermionic contributions to the constraints. A main result is that in spite of the presence of extrinsic curvature terms in the Hamiltonian, one still ends up with a hermitian contribution to $M$. In section 4 we put everything together, phrasing the Hamiltonian constraint as a Poisson bracket between the functional $M$ and the local volume functional $V(x)$. Then we quantize the theory, importing the results of [1], and construct elements of the physical Hilbert space $\mathbf{H}_{P h y s}$ satisfying all of the constraints. Section 5 is a summary and discussion section, including some potential areas for future investigation.

\section{PRELIMINARIES FOR THE HAMILTONIAN CONSTRAINT}

Before proceeding with the aims of this paper, let us first collect some relevant basic results and establish some notations and conventions. The starting Lagrangian density for the matter fields of this paper will be given by

$$
\begin{array}{r}
L=\frac{1}{4} \operatorname{det}\left({ }^{4} e\right) G_{\alpha \beta} F_{\mu \nu}^{\alpha} F_{\rho \sigma}^{\beta} g^{\mu \rho} g^{\nu \sigma} \\
+\frac{1}{2} \operatorname{det}\left({ }^{4} e\right) G_{I J} g^{\mu \nu}\left(D_{\mu} \phi^{I}\right)\left(D_{\nu} \phi^{J}\right)-\operatorname{det}\left({ }^{4} e\right) U\left(\psi^{r}, \psi_{r}^{\dagger}, \phi^{I}\right) \\
+\frac{1}{2}\left(\operatorname{det}\left({ }^{4} e\right) \psi_{r}^{\dagger} E_{A}^{\mu} \tau^{A} i \mathcal{D}_{\mu} \psi^{r}+\text { h.c. }\right) .
\end{array}
$$

It will be convenient, for the purposes of canonical decomposition into $3+1$ form, to put in place some conventions for labeling indices. Beginning Greek symbols $\alpha, \beta, \ldots$ will denote indices labeling the non-gravitational Yang-Mills gauge group $\mathcal{G}$, which for the conventional Standard Model is $S U(3) \otimes S U(2) \otimes U(1)$ or its extension to the gauge groups of Grand Unified Theories, whereas symbols from the middle $\mu, \nu, \ldots$ will refer to 4-dimensional spacetime indices. We will use symbols from the middle of the Latin alphabet $i, j, k, \ldots$ to denote 3 -dimensional spatial indices, and from the beginning $a, b, c \ldots$ to denote left-handed gravitational $S O(3, C)$ Lorentz indices, each set taking values $1,2,3$. So the Yang-Mills gauge connection and electric field will be given by $A_{i}^{\alpha}$, $\widetilde{\Pi}_{\alpha}^{i}$; and the Ashtekar variables, a $S L(2, C)$ connection and densitized triad are given by $A_{i}^{a}, \widetilde{E}_{a}^{i}$. [8] Upper case Latin letters $A, B, \ldots$ from the beginning of the alphabet denote $S O(3,1)$ Lorentz indices while Latin symbols from $I, J, \ldots$ will label the multiplet of Higgs fields, which transform in a given representation of $\mathcal{G}$. The corresponding phase space variables are given by $\phi^{I}, \widetilde{\pi}_{I}$. Indices $\alpha$ will be raised by $G^{\alpha \beta}$, suitable Killing-Cartan forms on $\mathcal{G}$, while indices $I$ will be lowered by the constant fiber metric $G_{I J}$ on the Higgs bundle. Finally, for the phase space variables of the two-component left-handed spin $1 / 2$ fermions $\psi^{r}, \tilde{\pi}_{r}$, symbols from the end of the Latin alphabet $r, s, \ldots$ will represent all the flavor indices, which can be extended to include all generations of fermions. To avoid cluttering the notation, spinorial indices shall be suppressed by using matrices and writing each Weyl fermion as a two-component single column matrix.

The Standard Model and Grand Unified Theories incorporate all fermionic particles as left-handed multiplet(s) of Weyl fermions transforming according to a certain representation (which we have denoted generically by $T$ ) of the Yang-Mills gauge group. The total covariant derivative for minimal coupling of fermions to gauge and gravitational forces is $\mathcal{D}_{i} \psi^{r}=\partial_{i} \psi^{r}-i A_{i}^{\alpha}\left(T_{\alpha}\right)_{s}^{r} \psi^{s}-i A_{i a} \frac{\tau_{a}}{2} \psi^{r}$, wherein $A_{i a}$ is precisely the Ashtekar (anti)self-dual spin connection gauging the local $S L(2, C)$ Lorentz symmetry [5], $\tau^{a=1,2,3}$ denote the standard Pauli matrices, and $\Gamma_{i a}$ denotes connection coefficients of the intrinsic Levi-Civita connection compatible with the triad $E_{a}^{i}$. It will also be convenient to define the following version of the fermionic covariant derivative $D_{i}^{\Gamma} \psi^{r}=\partial_{i} \psi^{r}-i A_{i}^{\alpha}\left(T_{\alpha}\right)_{s}^{r} \psi^{s}-i \Gamma_{i a} \frac{\tau^{a}}{2} \psi^{r}$, which subtracts out the extrinsic curvature part of $\mathcal{D}$. The Yang-Mills covariant derivative acts on the Higgs multiplet with representation $\mathcal{T}$ as $D_{i} \phi^{I}=\partial_{i} \phi^{I}-i A_{i}^{\alpha}\left(\mathcal{T}_{\alpha}\right)_{J}^{I} \phi^{J}$. Also of note is that the gravitational variables are singlets under the Yang-Mills gauge group and vice-versa. Finally, in this paper we will use the definition $G=\frac{8 \pi G_{N e w t o n}}{c^{3}}$.

\section{Results from the metric-ADM formalism}

Let us now set the stage by reviewing some of the main results of [3], which demonstrates the coupling of bosonic matter fields to metric-based gravity within an affine Lie-algebraic framework. In [3] the following integrals over 3 -space $\Sigma$ were defined

$$
V[n]=\int_{\Sigma} d^{3} x n \sqrt{h} ; \quad T[f]=\frac{2}{3 G} \int_{\Sigma} d^{3} x f \tilde{\pi}^{i j} h_{i j},
$$


where $\left(h_{i j}, \widetilde{\pi}^{i j}\right)$ are the spatial 3-metric and its conjugate momentum, and $n, f$ are arbitrary functions on $\Sigma$. Essentially, $V[n]$ is Misner's, and $T[f]$ is York's (integrated) time function, which have the following Poisson bracket

$$
\{T[f], V[n]\}=V[f n] .
$$

This implies that if $(\phi, \widetilde{\pi})$ are the canonical variables of any matter field whose energy density $\widetilde{\mu}=\sqrt{h} \mu$ does not depend on the gravitational ADM canonical momentum $\widetilde{\pi}^{i j}$, i.e. $\mu=\mu\left(h_{i j}, \phi, \widetilde{\pi}\right)$, then the matter part of the Hamiltonian constraint of the Einstein-matter system can be written as the pure Poisson bracket

$$
\{T[\mu], V[n]\}=\int_{\Sigma} d^{3} x n \mu \sqrt{h}
$$

This, together with the fact that the gravitational part of the Hamiltonian constraint can be recovered as the Poisson bracket of the imaginary part of the Chern-Simons functional $I_{C S}$ (built in the spinor representation from the Sen connection) and the $V[N]$ above [1 3 ], yield that the Hamiltonian constraint of the Einstein-matter system is the Poisson bracket of $\frac{1}{G^{2}} \operatorname{Im} I_{C S}+T[\mu]$ and $V[n]$. Here the matter field may be a phenomenological matter model, e.g. a fluid or a more general elastic material.

In [3] it was shown explicitly that (4) holds for any Yang-Mills-Higgs system, where the energy densities of the Yang-Mills and Higgs fields are (rewritten in density weight one form)

$$
\begin{aligned}
& \widetilde{\mu}_{Y M}=\frac{1}{2 \sqrt{h}} h_{i j}\left(G^{\alpha \beta} \widetilde{\Pi}_{\alpha}^{i} \widetilde{\Pi}_{\beta}^{j}+G_{\alpha \beta} \widetilde{B}^{\alpha i} \widetilde{B}^{\beta j}\right), \\
& \widetilde{\mu}_{H}=G^{I J} \frac{\widetilde{\pi}_{I} \widetilde{\pi}_{J}}{2 \sqrt{h}}+\frac{1}{2} \sqrt{h} h^{i j} G_{I J}\left(D_{i} \phi^{I}\right)\left(D_{j} \phi^{J}\right)+\sqrt{h} U,
\end{aligned}
$$

wherein $\widetilde{B}^{i \alpha}=\frac{1}{2} \widetilde{\epsilon}^{i j k} F_{j k}^{\alpha}$ denotes the magnetic field strength of $A_{i}^{\alpha}$. Here $U=U\left(\phi^{I}\right)$ is a scalar potential which can include the mass and self-interaction terms for the Higgs field, but is independent of the momenta of gravitational variables. Note that none of the terms in (5) depends on the extrinsic curvature of $\Sigma$, i.e. on the ADM canonical momentum $\widetilde{\pi}^{i j}$.

We will extend these results to the fundamental fields of the Standard Model, which entails generalization of the gravity-matter coupling to include spin $1 / 2$ fermions. Since a spin $1 / 2$ fermion constitutes a finite dimensional representation of the Lorentz group and not of the group of general coordinate transformations, a vierbein is needed in the description of fermionic coupling to gravity. An expression for $\widetilde{\mu}_{F}$ can be derived from the $3+1$ form of the standard Lagrangian for the Weyl spinor fields via the standard canonical formalism, or simply by rewriting the timetime component of the energy-momentum tensor for the Weyl spinor field (see, for instance, Ref. [4]) by identifying $\widetilde{\pi}_{r}=-i \sqrt{h} \psi_{r}^{\dagger}\left(n_{A} \tau^{A}\right)$ as the momentum conjugate to the two-component Weyl spinor $\psi^{r}\left(\widetilde{\pi}_{r}\right.$ is a two-component row vector in matrix notation if we write $\psi^{r}$ as a two-component column vector). Here $n_{A} \tau^{A}$ denotes the spinorial form of the future pointing unit timelike normal to (3-space) $\Sigma$ in the spacetime. The derivation of the fermionic contribution to the Hamiltonian constraint is carried out in Section 3, with the result

$$
\widetilde{\mu}_{F}=-\frac{1}{2 \sqrt{h}}\left(\widetilde{\pi}_{r} \widetilde{E}_{a}^{i} \tau^{a} \mathcal{D}_{i} \psi^{r}+\text { c.c. }\right) .
$$

Once fermions have been introduced into the theory, the potential can be extended $U \rightarrow U\left(\phi^{I}, \psi^{r}, \widetilde{\pi}_{s}\right)$ to include Higgs-fermion mass couplings, still remaining a pure algebraic expression of its arguments independent of $\widetilde{\pi}^{i j}$.

\section{Inclusion of fermions, and Ashtekar-ADM triad variables}

We will now proceed with the incorporation of fermions into the theory. Since a vierbein will be needed, we will transition directly into a nonmetric formulation of GR, where the triad replaces the metric as a fundamental degree of freedom. Since the fermionic energy density (6) depends on the extrinsic curvature, this poses an additional challenge in relation to its bosonic counterparts in writing the analogue of (4), which will need to be dealt with. Also, if one were to naively resort to the Ashtekar self-dual formalism in its original form, one would be confronted with issues of reality conditions unique to the existence of fermions. Both difficulties can be circumvented. The correspondence 
to an affine group structure is precise only if the generators are hermitian. As discussed in [1], for pure gravity this selects the imaginary part, $Q$, of the Chern-Simons functional rather the full Chern-Simons expression functional of the Ashtekar connection. Similarly, the corresponding hermitian part of the fermionic Hamiltonian density will be selected in the final expression of $M$ in (22), and the affine algebra derived is equivalent to the usual real total Hamiltonian constraint expressed in terms of the densitized triad variable and its conjugate momentum.

The fundamental extrinsic curvature and densitized triad ADM variables $\left(\widetilde{E}^{i a}, K_{i a}\right)$ have non-trivial Poisson bracket $\left\{\widetilde{E}^{i a}(x), K_{j b}(y)\right\}_{P . B .}=G \delta_{b}^{a} \delta_{j}^{i} \delta^{(3)}(x, y)$. To express (5) in terms of these variables, the pertinent entities in (5) can be expressed as

$$
\begin{gathered}
h=\operatorname{det}\left(h_{i j}\right)=\operatorname{det} \widetilde{E}=\frac{1}{3 !} \epsilon_{\sim} \epsilon_{k} \epsilon_{a b c} \widetilde{E}^{i a} \widetilde{E}^{j b} \widetilde{E}^{k c}, \\
h^{i j}=\frac{\widetilde{E}_{a}^{i} \widetilde{E}_{a}^{j}}{\operatorname{det} \widetilde{E}}, \quad h_{i j}=e_{\sim}^{a} e_{\sim j}^{a} \operatorname{det} \widetilde{E},
\end{gathered}
$$

where ${\underset{\sim}{i}}_{i}^{a}$ and ${\underset{\sim}{j}}_{j}^{a}$ are defined as the weight -1 inverse densitized triads and the imaginary part of the Chern-Simons functional of the Ashtekar connection, $Q$, can be expressed totally in terms of $\Gamma_{i a}$ (which depends only on the triad $e_{i}^{a}$ ) and the extrinsic curvature $K_{i a}$ when the substitution for $A_{i a}=i K_{i a}+\Gamma_{i a}$ is carried out in $Q$. The explicit expression is detailed in Appendix B of [1].

The density weight one version of the Hamiltonian constraint for gravity with cosmological constant $\Lambda$, with Higgs and spin $\frac{1}{2}$ Weyl fermionic multiplets minimally coupled to Yang-Mills fields is given by

$$
\widetilde{H}=\widetilde{H}^{g r a v}+\sqrt{h}\left(\mu_{H}+\mu_{Y M}+\mu_{F}\right)=0,
$$

wherein the respective Hamiltonian densities (the density weight zero form in the case of the matter contributions) are [9]

$$
\begin{aligned}
\widetilde{H}^{\text {grav }} & =\frac{1}{G^{2}}\left(\{Q, V(x)\}+\frac{G \Lambda}{2} V(x)\right) \\
\mu_{H} & =G^{I J} \frac{\widetilde{\pi}_{I} \widetilde{\pi}_{J}}{2(\operatorname{det} \widetilde{E})}+\frac{\widetilde{E}_{a}^{i} \widetilde{E}_{a}^{j}}{2(\operatorname{det} \widetilde{E})} G_{I J}\left(D_{i} \phi^{I}\right)\left(D_{j} \phi^{J}\right)+U\left(\phi^{I}, \psi^{r}, \widetilde{\pi}_{s}\right) ; \\
\mu_{Y M} & =\frac{1}{2} e_{\sim}^{a} e_{\sim}^{a}\left(G^{\alpha \beta} \widetilde{\Pi}_{\alpha}^{i} \widetilde{\Pi}_{\beta}^{j}+G_{\alpha \beta} \widetilde{B}^{\alpha i} \widetilde{B}^{\beta j}\right) ; \\
\mu_{F} & =-\frac{1}{h} \widetilde{\pi}_{r} \widetilde{E}_{a}^{i} \frac{\tau^{a}}{2}\left(\partial_{i} \psi^{r}+\left(K_{i b}-i \Gamma_{i b}\right) \frac{\tau^{b}}{2} \psi^{r}-i A_{i}^{\alpha}\left(T_{\alpha}\right)_{s}^{r} \psi^{s}\right)+h . c . \\
& =-\left\{\frac{1}{h} \widetilde{\pi}_{r} \widetilde{E}_{a}^{i} \frac{\tau^{a}}{2} D_{i}^{\Gamma} \psi^{r}+h . c .\right\}-\frac{i}{h} \widetilde{\pi}_{r} \epsilon^{a b c} \widetilde{E}_{a}^{i} K_{i b} \frac{\tau^{c}}{2} \psi^{r} .
\end{aligned}
$$

In the above, $Q$ is the imaginary part of the Chern-Simons functional of the Ashtekar connection $A_{i}^{a}$; and the expression of $\widetilde{H}^{g r a v}$ in this affine Lie-algebraic form is a key result of [1]. The fermionic Hamiltonian density $\widetilde{\mu}_{F}$ can be obtained from the hermitian action of fermions coupled to gravitational and Yang-Mills connections [6]. The resultant fermionic contribution to the Hamiltonian constraint indicated by $\widetilde{\mu}_{F}=\sqrt{h} \mu_{F}$ with $\mu_{F}$ as in (9) can be obtained by substituting the Ashtekar connection $A_{i a}=i K_{i a}+\Gamma_{i a}$ into the hermitian fermionic action[6].[10] This accounts for why $D_{i}^{\Gamma} \psi$ in the final line of (9) contains $\Gamma_{i a}$ but not $A_{i a}$.

It will also be useful to have the density weight one functional $T[f]$ in Ashtekar variables, given by the densitized trace of the extrinsic curvature

$$
T[1]=\frac{2}{3 G} \int_{\Sigma} d^{3} x K_{i}^{a} \widetilde{E}_{a}^{i}=\frac{2}{3 i G} \int_{\Sigma} d^{3} x\left(A_{i}^{a}-\Gamma_{i}^{a}\right) \widetilde{E}_{a}^{i} .
$$

The main result of [3] is that, including the bosonic matter with gravitational contributions, the local Hamiltonian constraint can be written as the Poisson bracket $\{M, V(x)\}$ for a suitably chosen functional $M$, in direct analogy to the first equation of (9) absent the cosmological constant term. 11] The present work aims to draw attention to the fact that the Hamiltonian constraint of the complete Standard Model or even Grand Unified Theories (GUTs) coupled to gravitation can be expressed in the form of an affine algebra with Hermitian generators. This, combined with the ability to construct coherent states using gauge invariant, diffeomorphism invariant group elements of the affine group, leads directly to the existence of elements of the physical Hilbert space $\mathbf{H}_{P h y s}$ of the coupled theory. These states thus form unitary, irreducible representations of the affine group $A f f(R)$. Moreover, the states implement at a quantum level the positivity of the spectrum of the volume operator. 


\section{FERMIONIC CONTRIBUTION TO THE CONSTRAINTS}

In this section, we derive the fermionic contribution to the super-Hamiltonian and kinematic constraints from the hermitian action of fermions coupled to gravitation and Yang-Mills theory; and determine the functional whose Poisson bracket with the volume element reproduces the former. To wit, the full multiplet of two-component lefthanded Weyl fields, $\psi^{r}$, coupled to gravity and unified Yang-Mills theory has the Hermitian action,

$$
\begin{aligned}
S_{W e y l}= & \frac{1}{2} \int d^{4} x\left(\operatorname{det}^{4} e\right) \psi_{r}^{\dagger} E_{A}^{\mu} \tau^{A} i \mathcal{D}_{\mu} \psi^{r}+h . c . \\
= & \frac{1}{2} \int d t \int d^{3} x(N e) \psi_{r}^{\dagger}\left(\mathcal{E}_{A}^{\mu}-n_{A} n^{\mu}\right) \tau^{A} i \mathcal{D}_{\mu} \psi^{r}+h . c . \\
= & \frac{1}{2}\left[\int d t \int d^{3} x N e \psi_{r}^{\dagger} \mathcal{E}_{A}^{\mu} \tau^{A} i \mathcal{D}_{\mu} \psi^{r}-\int d t \int d^{3} x e \psi_{r}^{\dagger}\left(n_{A} \tau^{A}\right) t^{\mu} i \mathcal{D}_{\mu} \psi^{r}\right. \\
& \left.+\int d t \int d^{3} x N^{\mu} e \psi_{r}^{\dagger}\left(n_{A} \tau^{A}\right) i \mathcal{D}_{\mu} \psi^{r}\right]+ \text { h.c. }
\end{aligned}
$$

In Eq.(11), the definition for $\tau^{A=0,1,2,3}$ is $\left(\tau^{0}=I_{2}, \tau^{a=1,2,3}=\right.$ Pauli matrices), and $\psi_{r}^{\dagger}=\bar{\psi}_{r}^{T}$ (the overline denotes complex conjugation). We first decomposed the vierbein as $E_{A}^{\mu}=\left(\varepsilon_{A}^{\mu}-n_{A} n^{\mu}\right)$, with the normal vector $n^{\mu}$ satisfying $n_{\mu} n^{\mu}=-1$ and $n_{A} \equiv n_{\mu} E_{A}^{\mu}$. In terms of the lapse and shift functions, the normal can be expressed as $n^{\mu}=\frac{\left(t^{\mu}-N^{\mu}\right)}{N}$, where $n_{\mu} N^{\mu}=0$. The fermion conjugate momentum is $\tilde{\pi}_{r}=\frac{\partial L}{\left(\partial £_{t} \psi^{r}\right)}=-i e \psi_{r}^{\dagger}\left(n_{A} \tau^{A}\right)$, wherein $£_{t}$ is the Lie derivative with respect to the vector field $t^{\mu} \partial_{\mu}=\left(N n^{\mu}+N^{\mu}\right) \partial_{\mu}$. Under the action of the Lorentz group, $\tilde{\pi}_{r}$ transforms as $\tilde{\pi}_{r}^{\prime}=\tilde{\pi}_{r} u_{L}^{-1}$ i.e. inversely as the left-handed spinor $\psi^{\prime r}=u_{L} \psi^{r} \quad \forall u_{L} \in S L(2, C)$. Carrying out a Legendre transformation into the Hamiltonian description, we can read off the spin $1 / 2$ contributions to the intial value constraints.

\section{The Hamiltonian constraint}

So the contribution to the Hamiltonian constraint, which has density weight one and $N$ as Lagrange multiplier, is thus

$$
\begin{aligned}
\widetilde{\mu}_{F}=\sqrt{h} \mu_{F} & =-\frac{1}{2} e \psi_{r}^{\dagger} \mathcal{E}_{A}^{\mu} \tau^{A} i \mathcal{D}_{\mu} \psi^{r}+\text { h.c. } \\
& =-\frac{1}{2} e \psi_{r}^{\dagger}\left(n_{B} \tau^{B}\right)\left(n_{C} \bar{\tau}^{C}\right) \mathcal{E}_{A}^{\mu} \tau^{A} i \mathcal{D}_{\mu} \psi^{r}+\text { h.c. } \\
& =\frac{1}{2} \widetilde{\pi}_{r}\left(n_{C} \mathcal{E}_{A}^{\mu}\right) \bar{\tau}^{C} \tau^{A} \mathcal{D}_{\mu} \psi^{r}+\text { h.c. }
\end{aligned}
$$

In the second line of Eq.(12), we have inserted the identity matrix $\left(n_{B} \tau^{B}\right)\left(n_{C} \bar{\tau}^{C}\right)=I_{2}$, with $\bar{\tau}^{A} \equiv\left(I_{2},-\tau^{1,2,3}\right)$. Furthermore, the entity $\left(n_{C} \mathcal{E}_{A}^{\mu}\right) \bar{\tau}^{C} \tau^{A}$ transforms according to the adjoint representation of $S L(2, C)$. It is spatial since $n_{\mu}\left(n_{C} \mathcal{E}_{A}^{\mu}\right) \bar{\tau}^{C} \tau^{A}=0$, and also traceless. From $n_{A} n^{A}=n_{\mu} n^{\mu}=-1$, the choice of $n_{A}=(-1,0,0,0)$ yields $\left(n_{C} \mathcal{E}_{A}^{\mu}\right) \bar{\tau}^{C} \tau^{A}=n_{C}\left(E_{A}^{\mu}+n_{A} E_{B}^{\mu} n^{B}\right) \bar{\tau}^{C} \tau^{A}=-E_{a}^{\mu} \tau^{a}$. Since $n_{\mu}\left(E_{a}^{\mu} \tau^{a}\right)=0$, a further choice of $n_{\mu}=-N \delta_{\mu}^{0}$ renders $E_{a}^{0}=0$. Thus, the fermionic contribution to the Hamiltonian constraint reduces to

$$
\begin{aligned}
\widetilde{\mu}_{F} & =-\widetilde{\pi}_{r} E_{a}^{i} \frac{\tau^{a}}{2} \mathcal{D}_{i} \psi^{r}+h . c . \\
& =-\widetilde{\pi}_{r} E_{a}^{i} \frac{\tau^{a}}{2}\left(\partial_{i} \psi^{r}+\frac{1}{4} A_{i A B} \bar{\tau}^{A} \tau^{B} \psi^{r}-i A_{i}^{\alpha}\left(T_{\alpha}\right)_{s}^{r} \psi^{s}\right)+h . c . \\
& =-\widetilde{\pi}_{r} E_{a}^{i} \frac{\tau^{a}}{2}\left(\partial_{i} \psi^{r}+\left(A_{i 0 b}+\frac{i}{2} \epsilon_{0 b c d} A_{i}^{c d}\right) \frac{\tau^{b}}{2} \psi^{r}-i A_{i}^{\alpha}\left(T_{\alpha}\right)_{s}^{r} \psi^{s}\right)+\text { h.c. } \\
& =-\frac{1}{\sqrt{h}} \widetilde{\pi}_{r} \widetilde{E}_{a}^{i} \frac{\tau^{a}}{2}\left(\partial_{i} \psi^{r}+\left(K_{i b}-i \Gamma_{i b}\right) \frac{\tau^{b}}{2} \psi^{r}-i A_{i}^{\alpha}\left(T_{\alpha}\right)_{s}^{r} \psi^{s}\right)+\text { h.c. }
\end{aligned}
$$

In the final step, we have denoted the spin connection $A_{i A B}$ by $K_{i a}=A_{i 0 a}$ and $\Gamma_{i a}=\frac{1}{2} \epsilon^{0 a b c} A_{i b c}$. In the absence of fermionic matter, $e_{(i}^{a} K_{j) a}$ is the extrinsic curvature. In general, $K_{i a}$ is the conjugate momentum to $\widetilde{E}^{i a}$ while $\Gamma_{i a}$ is the torsionless connection compatible with the triad.

We would like to construct a functional $M_{1 / 2}$ on the classical phase space such that

$$
\left\{M_{1 / 2}, V(x)\right\}_{P . B .}=\widetilde{\mu}_{F}(x) ;
$$


wherein the volume element is given by $V(x)=\sqrt{\frac{1}{3 !} \epsilon_{i j k} \epsilon^{a b c} \widetilde{E}_{a}^{i} \widetilde{E}_{b}^{j} \widetilde{E}_{c}^{k}}=\sqrt{\operatorname{det}(\widetilde{E})}$. To wit, the following identities are useful

$$
\begin{gathered}
\left\{V(x), K_{i a}(y)\right\}=\frac{G}{2} e_{i a}(x) \delta^{(3)}(x, y), \\
\left\{V(x), \int_{\Sigma} K_{i a} \widetilde{E}^{i a} d^{3} y\right\}=\frac{3 G}{2} V(x) .
\end{gathered}
$$

Due to the hermitization, the term containing $K_{i a}$ in (13) is just $-i \epsilon^{a b c} \widetilde{E}_{a}^{i} K_{i b}\left(\widetilde{\pi}_{r} \tau_{c} \psi^{r}\right)$ which has vanishing Poisson bracket with $V(x)$. It follows that $\widetilde{\mu}_{F}$ commutes with $V(x)$; and a solution for $M_{1 / 2}$ is

$$
\begin{array}{r}
M_{1 / 2}=T\left[-\mu_{F}\right]=\int d^{3} y\left(\frac{2 \widetilde{K}}{3 G \operatorname{det}(\widetilde{E})}\right)\left\{\widetilde { \pi } _ { r } \widetilde { E } _ { a } ^ { i } \frac { \tau ^ { a } } { 2 } \left(\partial_{i} \psi^{r}+\left(K_{i b}-i \Gamma_{i b}\right) \frac{\tau^{b}}{2} \psi^{r}\right.\right. \\
\left.\left.-i A_{i}^{\alpha}\left(T_{\alpha}\right)_{s}^{r} \psi^{s}\right)+ \text { h.c. }\right\} ;
\end{array}
$$

wherein $\tilde{K} \equiv K_{i a} \tilde{E}^{i a}$, and $\frac{2}{3 G} \int \tilde{K} d^{3} y=T[1]$ from (10). Note that the integrand in $M_{1 / 2}$ is of density weight one and thus $M_{1 / 2}$ is diffeomorphism-invariant, and it is moreover classically real since we started from the Hermitian Weyl action (11).

Similarly, the solutions for the Higgs and Yang-Mills contributions are

$$
\left\{M_{0}, V(x)\right\}_{P . B .}=\widetilde{\mu}_{H}(x), \quad\left\{M_{1}, V(x)\right\}_{P . B .}=\widetilde{\mu}_{Y M}(x),
$$

and

$$
M_{0}=T\left[-\mu_{H}\right], \quad M_{1}=T\left[-\mu_{Y M}\right] .
$$

Replacing $i K_{i a}+\Gamma_{i a}$ by $A_{i a}$ achieves the alternative to express 12] $M_{0}, M_{1 / 2}, M_{1}$ in terms of Ashtekar variables $\left(\widetilde{E}^{i a}, A_{i a}\right)$; but it should be noted that unlike Ashtekar's totally chiral prescription [5], the hermitian conjugate operation in the action and Hamiltonian involves the spin connection of the opposite chirality. Thus spin connections of both chiralities $\pm i K_{i a}+\Gamma_{i a}$ are needed to render $M_{1 / 2}$ real.

\section{The kinematic constraints}

We have written the fermionic contribution to the Hamiltonian constraint as a Poisson bracket involving the functional $M_{1 / 2}$. We will next derive the spin $1 / 2$ contributions to the kinematic constraints. From the third term on the right hand side of (11), one has

$$
-\frac{1}{2} \int d t \int d^{3} x N^{\mu} e \psi_{r}^{\dagger}\left(n_{A} \tau^{A}\right) i \mathcal{D}_{\mu} \psi^{r}+\text { h.c. }=\frac{1}{2} \int d t \int d^{3} x \widetilde{\pi}_{r} N^{\mu} \mathcal{D}_{\mu} \psi^{r}+\text { h.c. }
$$

Stipulating that $n_{\mu} N^{\mu}=0$, then in the chosen gauge the shift vector is purely spatial and one can make the identification $N^{\mu} \rightarrow N^{i}$, which implies the fermionic contribution to the smeared vector constraint

$$
H_{i}^{W e y l}\left[N^{i}\right]=\frac{1}{2} \int d^{3} x N^{i} \widetilde{\pi}_{r} \mathcal{D}_{i} \psi^{r}+\text { h.c. }
$$

The second term of (11) yields

$$
\begin{array}{r}
\frac{1}{8} \int d t \int d^{3} x e \psi_{r}^{\dagger}\left(n_{A} \tau^{A}\right) i t^{\mu} A_{\mu A B} \bar{\tau}^{A} \tau^{B} \psi^{r}+h . c . \\
=-\frac{1}{2} \int d t \int d^{3} x \widetilde{\pi}_{r}\left(\frac{\tau^{c}}{2}\right) \psi^{r} t^{\mu}\left(A_{\mu 0 c}+\frac{i}{2} \epsilon_{0 c a b} A_{\mu}^{a b}\right)+h . c .
\end{array}
$$

where $(-i) t^{\mu}\left(A_{\mu 0 c}+\frac{i}{2} \epsilon_{0 c a b} A_{\mu}^{a b}\right)$ is a Lagrange multiplier. So the fermionic contribution to the smeared gravitational Gauss' law constraint is

$$
G_{c}^{W e y l}\left[\eta^{c}\right]=-\int d^{3} x \eta^{c}\left[i \widetilde{\pi}_{r}\left(\frac{\tau^{c}}{2}\right) \psi^{r}\right]
$$


for some complex $\mathrm{SO}(3, \mathrm{C})$-valued parameter $\eta^{c}(x)$. Similarly, one can read off the analogous Yang-Mills part

$$
\frac{1}{2} \int d t \int d^{3} x e \psi_{r}^{\dagger}\left(n_{A} \tau^{A}\right) i t^{\mu}\left(-i A_{\mu}^{\alpha}\left(T_{\alpha}\right)_{s}^{r} \psi^{s}\right)+\text { h.c. }=\frac{i}{2} \int d t \int d^{3} x t^{\mu} A_{\mu}^{\alpha} \widetilde{\pi}_{r}\left(T_{\alpha}\right)_{s}^{r} \psi^{s}+\text { h.c. },
$$

where $t^{\mu} A_{\mu}^{\alpha}$ is the corresponding Lagrange multiplier. So we can extract the fermionic contribution to the smeared Yang-Mills Gauss' law constraint, for some $\mathcal{G}$-valued real parameter $\eta^{\alpha}(x)$, given by

$$
G_{\alpha}^{W e y l}\left[\eta^{\alpha}\right]=\int d^{3} x \eta^{\alpha}\left[\widetilde{\pi}_{r}\left(i T_{\alpha}\right)_{s}^{r} \psi^{s}\right]
$$

In gravitational theories incorporating local gauge-invariance, the gauge-invariant vector (super-momentum) constraint, with the shift function as Lagrange multiplier, generates gauge-covariant Lie derivatives rather than pure spatial Lie derivatives. The constraint itself is gauge-invariant under all local gauge symmetries of the theory. By adding suitable combinations of the Gauss' Law constraints generating local gauge symmetries, a non-gauge-invariant corresponding constraint can be constructed to generate true spatial Lie derivatives of the dynamical variables. It follows that any spatial integral over the entire Cauchy surface of any tensor density weight one gauge-invariant composite of dynamical variables will be invariant under both spatial diffeomorphisms and also under local gauge symmetries generated by the Gauss' Law constraints. Thus the $M^{\text {Grav }}, M_{0}, M_{1}, M_{1 / 2}$ in Eq. (22) entities that we construct later on will all be gauge and spatial diffeomorphism invariant as they are integrals of weight one tensor densities of gauge-invariant composites of dynamical variables.

\section{PUTTING IT ALL TOGETHER}

We have addressed all of the ingredients necessary to construct a theory which respects the Hamiltonian constraint as well as the kinematic constraints for gravity coupled with the matter fields of the Standard Model in the AshtekarADM triad variables. So we may now proceed with a view toward quantization of the theory and the construction of elements of the physical Hilbert space $\mathbf{H}_{\text {Phys }}$. Putting everything together, recall that $\widetilde{H}=\widetilde{H}^{g r a v}+\widetilde{\mu}_{H}+\widetilde{\mu}_{F}+\widetilde{\mu}_{Y M} \cdot[13]$ We can define the following contributions to the functional $M=M^{G r a v}+G^{2}\left(M_{0}+M_{1 / 2}+M_{1}\right)$ with

$$
\begin{aligned}
& M^{\text {Grav }}=Q \\
& M_{0}=T\left[-\mu_{H}\right] \\
& M_{1}=T\left[-\mu_{Y M}\right], \\
& M_{1 / 2}=\int d^{3} y\left[\frac{2 \widetilde{K}}{3 G \operatorname{det}(\tilde{E})}\right]\left\{\widetilde{\pi}_{r} \widetilde{E}_{a}^{i} \frac{\tau^{a}}{2} \mathcal{D}_{i} \psi^{r}+\text { h.c. }\right\}=T\left[-\mu_{F}\right]
\end{aligned}
$$

with $T[f(x)] \equiv \frac{2}{3 G} \int d^{3} x f K_{a i} \tilde{E}^{a i}$ and $\widetilde{K}=K_{i}^{a} \widetilde{E}_{a}^{i}$

Then the Hamiltonian constraint of the Standard Model coupled to gravity can be written as a Poisson bracket

$$
\{M, V(x)\}+\frac{G \Lambda}{2} V(x)=0 .
$$

The full Hamiltonian constraint for gravity coupled with matter fields, in direct analogy to [1], can be written as an affine Lie algebra. Since all terms of $M$ are real, then their quantum versions will correspond to Hermitian operators. Upon making the replacement $Q \rightarrow M$ in [1] and carrying out the steps for quantization, then the Hamiltonian constraint becomes the affine Lie algebra

$$
\widehat{H}(x)|\psi\rangle=[i \widehat{M}, \widehat{V}(x)]|\psi\rangle+\lambda \widehat{V}(x)|\psi\rangle=0 ; \quad \lambda=\frac{{ }^{\sim} G \Lambda}{2} .
$$

So the solution to the quantum Hamiltonian constraint (24) is tantamount to the statement that $|\psi\rangle$ must form a representation space of the affine group $A f f(R)$. But for $|\psi\rangle$ to be a physical state, it must additionally be gauge invariant 14] and diffeomorphism invariant

$$
\widehat{H}_{i}(x)|\psi\rangle=\widehat{G}_{a}(x)|\psi\rangle=\widehat{G}_{\alpha}(x)|\psi\rangle=0
$$

Let $|\eta\rangle=|0,0\rangle \in \operatorname{Ker} \widehat{H}(x)$ be a gauge invariant, diffeomorphism invariant and normalized fiducial state. We would like to construct gauge invariant, diffeomorphism-invariant states $|a, b\rangle \in \operatorname{Ker} \widehat{H}_{i}(x), \widehat{G}_{a}(x), \widehat{G}_{\alpha}(x)$ as elements of 
the physical Hilbert space $\mathbf{H}_{\text {Phys }}$ from affine group elements, for spatially homogeneous quantities $a$ and $b$ of mass dimensions $[a]=0$ and $[b]=3$, given by

$$
|a, b\rangle=e^{-i a \widehat{M}} e^{-i b \widehat{V}}|0,0\rangle \in \mathbf{H}_{P h y s}
$$

But for this to be the case, the generators $\widehat{M}$ and $\widehat{V}$ must be gauge-diffeomorphism invariant. The 3-space volume operator $\widehat{V}$ is already gauge-diffeomorphism invariant since it is the integral of a geometric object of density weight one $\int_{\Sigma} d^{3} x \widehat{V}(x)$, whose internal indices are completely contracted. For the object $\widehat{M}$, it suffices to note via density weight counting, that the integrand of $\widehat{M}$ is an object of density weight one, hence $\left[\widehat{M}, \widehat{H}_{i}\right]=0$ and $M$ is diffeomorphism invariant. Indeed it is the convolution of $T$, which is of density weight one on account of the densitized triad $\widetilde{E}_{a}^{i}$, and the various contributions to $\mu$ which are of density weight zero. Additionally, $M$ is gauge invariant (e.g. $\left[\widehat{M}, \widehat{G}_{a}\right]=\left[\widehat{M}, \widehat{G}_{\alpha}\right]=0$ ) since it is constructed from tensorial geometric objects whose gauge indices (gravitational and nongravitational) are completely contracted. The result is that $|a, b\rangle \in \mathbf{H}_{P h y s}$ constitute a set of physical states for the full theory of the Standard Model coupled to gravity, through unitary irreducible representations of the affine group, in direct analogy with the results of [1] .

The affine algebra for gravity coupled with the Standard Model implies a fundamental uncertainty relation

$$
\frac{\Delta V}{\langle V\rangle} \Delta M \geq 2 \pi \Lambda L_{\text {Planck }}^{2}
$$

wherein $V$ is the total volume of the universe. This is an extension of the uncertainty relation between $Q$ and $V$ obtained in [1], which depends on the cosmological constant; and equation (27) is a result of treating gravity and matter on equal quantum footing. It is intriguing that current observations place $\Lambda L_{\text {Planck }}^{2} \sim 10^{-120}$ which renders the uncertainty small, but nevertheless non-vanishing, while the precise correspondence of the Hamiltonian constraint to an affine algebra is predicated upon a non-vanishing cosmological constant. Affine algebraic quantization of both gravitation and matter on equal footing may yield a new perspective on the cosmological constant problem.

\section{SUMMARY AND FUTURE RESEARCH}

This work demonstrates that a complete description of the interaction of matter and all forces (gravitational and non-gravitational) can in fact be realized within a quantum affine algebraic framework. It has been shown that the generalization of the affine group formalism for four dimensional, Lorentzian signature quantum gravity in the Ashtekar variables in [1] straightforwardly extends to the coupling to non-gravitational fields [15]. We have utilized this framework to construct elements of the physical Hilbert space of the Standard Model coupled to gravity.

The construction of a physical Hilbert space for gravity has until now remained an unsolved problem in theoretical physics, and the main contribution of this paper has been to provide a glimpse of certain elements of this space. The phenomenological implications of this result opens up various areas for further investigation, not limited to and including: (i) To check for consistency with symmetry breaking via the Higgs mechanism, using the affine coherent states $|a, b\rangle$ as the starting point. (ii) To examine the quantum gravitational effects encoded in and induced from the states $|a, b\rangle$ in the semiclassical limit below the Planck scale, and to compare its predictions with those of quantum field theory on curved spacetime. It is conceivable that in this limit, imprints of the Planck scale physics could in some sense be probed via this formalism.

\section{ACKNOWLEDGEMENTS}

This work has been supported in part by the Office of Naval Research under Grant No. N-000-1412-WX-30191, the National Science Council of Taiwan under Grant No. NSC 101-2112-M-006 -007 -MY3, and the National Center for Theoretical Sciences, Taiwan.

* Electronic address: 12897107@mail.ncku.edu.tw

† Electronic address: ita@usna.edu

‡ Electronic address: cpsoo@mail.ncku.edu.tw 
[1] Chou Ching-Yi, Eyo Ita and Chopin Soo, 'Affine group representation formalism for four dimensional, Lorentzian, quantum gravity' Class. Quantum Grav. 30 (2013): 065013

[2] László B. Szabados, 'On the role of conformal three geometries in the dynamics of General Relativity', Class. Quantum Grav. 19 (2002) 2375-2392

[3] László B. Szabados, 'A note on the Hamiltonian constraint in canonical GR', Class. Quantum Grav. 25 (2008): 095005

[4] R. Penrose, W. Rindler, 'Spinors and spacetime', vol.2 (Cambridge University Press, 1986)

[5] A. Ashtekar, 'Lectures on non-perturbative canonical gravity' (World Scientific, 1991)

[6] T. Thiemann, 'Modern Canonical Quantum General Relativity' (Cambridge University Press, 2007)

[7] A. Ashtekar, J,Romano, and R. Tate 'New variables for gravity: Inclusion of matter', Phys. Rev. D40, 2572-2587 (1989)

[8] We will often utilize the tilde to denote objects of density weight one. We will omit the tilde for the local volume element $V(x)$ to avoid cluttering the notation. Its density weight will be clear from the context.

[9] The matter Hamiltonian densities are of mass dimension $[\mu]=4$, hence the factor of $\frac{1}{G^{2}}$ which multiplies the dimensionless $Q$. This balances the mass dimensions of the gravitational with matter terms in accordance with the Einstein equations.

[10] The derivation is provided in the next section.

[11] It is crucial that the functional $M$ be chosen so as to produce the density weight one form of the Hamiltonian constraint. $M$ is thus diffeomorphism invariant. Moreover, as noted in [1, [6], only for this density weight does one have a constraint with good self-regularizing properties, which is free of ultraviolet divergences.

[12] The subscripts on $M$ refer to the spins of the respective contributions to $M$.

[13] where $\widetilde{H}^{\text {grav }}=\frac{1}{G^{2}}\left(\{Q, V(x)\}+\frac{G \Lambda}{2} V(x)\right),\left\{M_{0}, V(x)\right\}=\widetilde{\mu}_{H},\left\{M_{1 / 2}, V(x)\right\}=\widetilde{\mu}_{F}$, and $\left\{M_{1}, V(x)\right\}=\widetilde{\mu}_{Y M}$.

[14] That is, gauge invariant with respect to the gravitational and the nongravitational Yang-Mills gauge groups.

[15] Generalization to supersymmetric extensions of the Standard Model should be straightforward via the techniques of this paper, and application to supergravity and GUTs can as well be examined. 